Relations industrielles

Industrial Relations

\title{
Wage Formation in Europe par Emmanuel MERMET, Bruxelles : European Trade Union Institute, 2001, 182 p., ISBN 2-930143-93-2.
}

\section{Evelyne Léonard}

Volume 58, numéro 4, automne 2003

URI : https://id.erudit.org/iderudit/007826ar

DOI : https://doi.org/10.7202/007826ar

Aller au sommaire du numéro

Éditeur(s)

Département des relations industrielles de l'Université Laval

ISSN

0034-379X (imprimé)

1703-8138 (numérique)

Découvrir la revue

Citer ce compte rendu

Léonard, E. (2003). Compte rendu de [Wage Formation in Europe par Emmanuel MERMET, Bruxelles : European Trade Union Institute, 2001, 182 p., ISBN 2-930143-93-2.] Relations industrielles / Industrial Relations, 58(4),

712-714. https://doi.org/10.7202/007826ar

Tous droits réservés (c) Département des relations industrielles de l'Université Laval, 2003
Ce document est protégé par la loi sur le droit d'auteur. L’utilisation des services d’Érudit (y compris la reproduction) est assujettie à sa politique d'utilisation que vous pouvez consulter en ligne.

https://apropos.erudit.org/fr/usagers/politique-dutilisation/ 


\section{Wage Formation in Europe}

par Emmanuel MERMET, Bruxelles : European Trade Union Institute, 2001, 182 p., ISBN 2-930143-93-2.

Il ne faut pas s'attendre à trouver dans cet ouvrage une analyse théorique approfondie des mécanismes de formation des salaires aujourd'hui en Europe. $\mathrm{Au}$ contraire, cette publication éditée par l'Institut syndical européen (ISE/ETUI), qui fait partie de la Confédération européenne des syndicats (CES/ETUC), poursuit d'abord des objectifs descriptifs et politiques au sens large. Avec pour projet de contribuer à la coordination des négociations salariales au plan européen, l'ouvrage se donne comme tâche d'analyser ce phénomène multidimensionnel qu'est la formation des salaires en Europe aujourd'hui, d'établir un état de la situation et de répondre à plusieurs questions : quel est l'impact de l'introduction de la monnaie unique européenne sur la négociation collective dans chaque pays ? Quel pourrait être le rôle d'une coordination européenne de la négociation ? Est-il possible de remplacer la modération salariale qui a prévalu pendant longtemps dans de nombreux pays par une approche plus dynamique des politiques salariales ?

Abordant la formation des salaires sous l'angle économique, l'ouvrage comprend deux parties. La première présente une analyse comparée des enjeux actuels de la formation des salaires en Europe, la seconde est composée de brefs chapitres nationaux portant sur les quinze États membres de l'Union au moment de la publication ainsi que la Norvège. Les analyses développées par Mermet conduisent en finale à des recommandations de la CES sur la coordination de la négociation collective à l'échelon européen, qui sont jointes en annexe.

La première partie, qui constitue l'analyse comparative, s'attache surtout aux enjeux soulevés par l'introduction de l'euro. Elle comprend six sections. L'auteur commence par un récapitulatif succinct des principales théories sur la formation des salaires et une analyse de la composition des coûts du travail. Il enchaîne par l'étude des effets induits par l'introduction de l'euro sur les processus de formation des salaires. Suit l'examen de la situation des salaires dans l'Union européenne. Ensuite, les systèmes nationaux de négociation collective sont abordés, sous l'angle du niveau de négociation des salaires, des déterminants des revendications salariales et des calendriers de négociation. Mermet s'engage enfin, dans la cinquième section du chapitre comparatif, dans des propositions pour une coordination de la négociation salariale en Europe, qui prendrait la forme d'une ligne de conduite formulée annuellement par la CES à l'intention des organisations syndicales nationales qui en sont membres. Cette ligne de conduite prendrait en compte l'inflation et la productivité comme principaux déterminants des revendications salariales. Une conclusion clôture cette partie en insistant sur le processus de pilotage qui serait nécessaire pour accompagner une telle coordination.

La seconde partie comprend les chapitres nationaux. Son introduction précise utilement que l'information fournie sur chaque pays provient des réponses des organisations affiliées à la CES à un questionnaire qui leur avait été envoyé. Tous les chapitres nationaux respectent une structure identique : système de négociation collective, importance de la thématique salariale dans l'ensemble de la négociation, rôle des confédérations nationales dans la négociation salariale, déterminants des revendications salariales, etc.

Un regard critique sur l'ouvrage fait d'abord apparaître que le double objectif d'analyse de la situation et de proposition afin de coordonner les négociations 
entre pays peut conduire à une certaine confusion : les registres analytique et normatif s'entrecroisent dans certains chapitres, tandis que le projet d'une coordination accrue des négociations salariales est posé a priori comme souhaitable.

La section récapitulant les principaux apports théoriques sur la formation des salaires ne satisfera sans doute pas le spécialiste scientifique du domaine tant elle est condensée et, par conséquent, assez superficielle. Il manque d'ailleurs dans la bibliographie plusieurs des références évoquées. Elle a pourtant le mérite de montrer en quoi la formation des salaires ne peut être analysée qu'à partir du moment où les théories prennent en compte le rôle des institutions. Tant que le salaire reste compris comme le produit du marché ou comme le résultat de l'exploitation du travail par le capital, sa formation en tant que telle est délicate à analyser. Mermet montre bien le basculement que permettent des approches économiques récentes qui permettent de comprendre en tant que tel le processus de formation des salaires, avec le rôle qu'y jouent les institutions.

D'un point de vue théorique, la question des déterminants des revendications salariales semble quelque peu ambiguë, manquant d'ailleurs d'une réflexion conceptuelle préalable. Les revendications salariales sont ainsi envisagées en tant que résultat d'un nombre réduit de « déterminants » économiques, comme l'inflation et la productivité. Ces variables sont considérées d'office comme déterminants des revendications, et non pas comme arguments, le raisonnement laissant alors peu de place aux stratégies et tactiques de négociation mises en œuvre par les acteurs. Par ailleurs, d'autres déterminants tels que l'équité ou l'égalité, par exemple, sont totalement ignorés.

En ce qui concerne les chapitres nationaux, inévitablement dans ce genre d'exercice, l'enchaînement des seize synthèses, même brèves, comptant chacune entre quatre et douze pages, - ce qui est en soi une performance à souligner - , ne peut qu'être fastidieux, et toutes sont fondées sur des sources secondaires. Le lecteur y trouvera cependant, sous une forme remarquablement synthétique, une présentation systématique des principales caractéristiques des négociations salariales dans seize pays, mise en perspective avec l'analyse des dynamiques européennes. En ce sens, l'ouvrage offre une mine d'information très appréciable.

La question de la coordination des négociations, au cœur du projet même de l'ouvrage, est centrale et on comprend en quoi elle interpelle une organisation syndicale supranationale comme la CES. Elle est clairement documentée par l'ouvrage, bien défendue et étoffée par l'auteur. On peut toutefois regretter qu'il manque une réflexion sur la faisabilité d'une telle coordination, non pas en termes de capacité de la CES à l'organiser, mais bien en termes de capacité, ou de volonté politique, des organisations syndicales nationales à adopter la ligne de conduite proposée par la CES et à la mettre en œuvre dans leurs propres négociations. Le lecteur reste également sur sa faim quant à d'autres enjeux majeurs qui sont liés à la négociation salariale et à la formation des salaires, et en particulier le problème de l'emploi et les questions de solidarité et d'équité. La question du chômage, par exemple, est expédiée très rapidement. Or, si la modération salariale qui a prévalu pendant une dizaine d'années en Europe est en partie liée à l'introduction de la monnaie unique, elle est aussi en lien avec les politiques d'emploi et les objectifs de réduction du chômage, dans lesquels les interlocuteurs sociaux ont un rôle à jouer. Il aurait été intéressant d'aborder cette question.

Cela dit, l'ouvrage fournit incontestablement un examen documenté et actualisé sur la formation des salaires en Europe. Il atteint donc ses objectifs, qui 
étaient d'établir un état de la question et de contribuer à la construction d'une coordination. Il faut en outre souligner que cette question de la coordination est non seulement d'une actualité brûlante, mais qu' elle renvoie à d'autres qui constituent autant d'enjeux clés pour les relations industrielles dans l'Union européenne aujourd'hui : conséquences de l'introduction de l'euro sur les relations industrielles ; pertinence de politiques de modération salariale ; question récurrente de la convergence des systèmes nationaux de relations industrielles. En ce sens, cet ouvrage apporte une contribution très pertinente à l'étude des relations professionnelles européennes.

EVELYNE LÉONARD

Université catholique de Louvain

\section{L'Europe syndicale au quotidien : la représentation des salariés en France, Allemagne, Grande-Bretagne et Italie}

par Christian Dufour et Adelheid Hege, Bruxelles : Presses interuniversitaires européennes, 2002, 256 p., ISBN 90-5201-918-5.

Dans leur ouvrage, Dufour et Hege brossent un tableau des pratiques au chapitre de la représentation syndicale en vue de déterminer quels modèles nationaux sont les plus à même de répondre aux défis posés par l'internationalisation. Cette étude comparative propose de vérifier empiriquement les rôles des représentants compte tenu des cadres institutionnels dans lesquels l'acte représentatif prend forme.

L'ouvrage se scinde en deux grandes parties. La première, les chapitres 1 à 4 , est composée de quatre monographies descriptives. Les enquêtes sur le terrain au sein de quatre pays : la France, l'Allemagne, la Grande-Bretagne et l'Italie, se sont déroulées entre 1990 et 1995 dans 15 sites par pays. Pour les fins de l'ouvrage, les auteurs ont retenu un cas par pays. Des entretiens menés auprès des membres des équipes de représentants fournissent une description riche $\mathrm{du}$ fonctionnement des structures institutionnelles et organisationnelles et un portrait détaillé du travail quotidien effectué par les représentants. Les chapitres 1 et 2 offrent un panorama d'un établissement en France et d'un en Allemagne, deux pays à système de représentation dual où le formalisme imprègne les instances de représentation. Les chapitres 3 et 4 décrivent deux systèmes monistes de représentation, ceux de la Grande-Bretagne et de l'Italie. Le volontarisme et la loi du free collective bargaining caractérisent le système anglais alors que l'Italie expérimente depuis 1994 de nouvelles structures de représentation initiées par les organisations syndicales, les RSU (rappresentaza sindacale unitaria). Dès cette première section, les auteurs lèvent le voile sur ce qui est commun aux quatre pays : le rôle des représentants qui dépasse amplement celui prescrit par leurs cadres institutionnels respectifs.

La seconde partie, davantage analytique, fait état des points de convergence et de divergence entre les pays étudiés en regard des conditions d'exercice de la représentation au-delà des cadres institutionnels. L'ouvrage se penche d'abord sur les différences entre les systèmes de représentation au plan des structures nationales et des particularités qui les sous-tendent. Par la suite, une analyse plus poussée dévoile les écarts quant aux caractéristiques de la représentation locale. C'est d'ailleurs un des aspects qui confère à l'ouvrage son originalité. Trop souvent, les études comparatives se limitent à l'examen des structures nationales et à celui des formes institutionnelles, occultant ainsi la base d'une réelle comparaison internationale. L'angle d'analyse de Dufour et Hege privilégie une approche plus sociologique qui permet d'appréhender le rôle prépondérant des représentants et de 